\title{
SCHUR Q-FUNCTIONS AND SPIN CHARACTERS OF SYMMETRIC GROUPS I
}

\author{
A. O. Morris* and A. A. Abdel-Aziz ${ }^{\dagger}$ \\ Dedicated to Dominique Foata on his 60th birthday
}

Submitted: March 31, 1995; Accepted: November 15, 1995

\section{Introduction}

In a classic paper, I. Schur [6] introduced a class of symmetric functions, now called Schur $Q$ functions, in order to determine the irreducible spin (projective) characters of symmetric groups. In the case of the ordinary characters of symmetric groups, going back to the early work of D. E. Littlewood and A. R. Richardson [3], the corresponding Schur functions have been used to give useful combinatorial formulae for determining explicit values for these characters (see for example [2]). Our aim in this paper is to obtain combinatorial formulae for the spin characters. We see that as always in the spin case, the formulae are considerably more complicated with a number of new interesting phenomena arising.

\section{Notation and Preliminaries}

\subsection{Compositions and partitions}

If $\ell$ is a positive integer, then $\lambda=\left(\lambda_{1}, \ldots, \lambda_{m}\right)$ is a composition of $\ell$ if $\lambda_{i}(i=1, \ldots, m)$ are positive integers such that

$$
\sum_{i=1}^{m} \lambda_{i}=\ell .
$$

If, in addition

$$
\lambda_{1} \geq \lambda_{2} \geq \ldots \lambda_{m}>0
$$

then $\lambda$ is a partition of $\ell ; \lambda$ is called a strict partition if

$$
\lambda_{1}>\lambda_{2}>\ldots \lambda_{m}>0 .
$$

When necessary, we write $\ell(\lambda)$ for $m$, the length of $\lambda$ and $|\lambda|$ for $\ell$, the weight of $\lambda$. Let $P(\ell)$ denote the set of partitions of $\ell, S P(\ell)$ the set of strict partitions of $\ell$ and $O(\ell)$ the set of partitions where

${ }^{*}$ Department of Mathematics, University of Wales, Aberystwyth, Dyfed SY23 3BZ

${ }^{\dagger}$ Department of Mathematics, Faculty of Education in Abha, PO Box 9002, Abha, Saudi Arabia 
all the parts $\lambda_{i}$ are odd. Also, we write $\lambda \backslash\left\{\lambda_{i}\right\}$ for the partition $\left\{\lambda_{1}, \ldots, \lambda_{i-1}, \lambda_{i+1}, \ldots, \lambda_{m}\right\}$, that is, with the $i$ th part deleted with an obvious extension to the deletion of more parts.

Partitions are also denoted as $\lambda=\left(1^{m_{1}} 2^{m_{2}} \ldots\right)$, indicating that the part $i$ is repeated $m_{i}$ times.

If $\lambda \in P(\ell)$, let

$$
U(\lambda)=\left\{\mu \in P(|\mu| \leq \ell) \mid\left\{\mu_{1}, \ldots, \mu_{\ell(\mu)}\right\} \subseteq\left\{\lambda_{1}, \ldots, \lambda_{m}\right\}\right.
$$

that is, $U(\lambda)$ is the set of sub-partitions of $\lambda$. For $r \leq \ell$, let

$$
U(\lambda, r)=\{\mu \in U(\lambda) \mid \mu \in P(r)\},
$$

$u(\lambda)=|U(\lambda)|, u(\lambda, r)=|U(\lambda, r)|$. Then, clearly $U(\lambda, \ell)=\{\lambda\}$ and $u(\lambda) \leq 2^{\ell(\lambda)}$ and $u(\lambda)=2^{\ell(\lambda)}$ if and only if $\lambda \in S P(\ell)$.

Definition 2.1 A separation of $\lambda \in P(\ell)$ corresponding to the composition $w=\left(w_{1}, w_{2}, \ldots, w_{k}\right)$ of $\ell$ is a $k$-set of partitions $\left(\lambda^{(1)}, \lambda^{(2)}, \ldots, \lambda^{(k)}\right)$ such that $\lambda^{(i)} \in P\left(w_{i}\right)(i=1, \ldots, k)$.

A complete set of separations $\left(\lambda^{(1)}, \ldots, \lambda^{(k)}\right)$ of $\lambda$ corresponding to the composition $w=$ $\left(w_{1}, \ldots, w_{k}\right)$ can be obtained by the following algorithm.

$$
\lambda^{(1)} \in U\left(\lambda, w_{1}\right), \lambda^{(2)} \in U\left(\lambda \backslash \lambda^{(1)}, w_{2}\right), \ldots, \lambda^{(k)} \in U\left(\lambda \backslash\left(\lambda^{(1)}, \ldots, \lambda^{(k-1)}\right), w_{k}\right)
$$

where

$$
U(\lambda) \supset U\left(\lambda \backslash \lambda^{(1)}\right) \supset U\left(\lambda \backslash\left(\lambda^{(1)}, \lambda^{(2)}\right)\right) \supset \ldots \supset U\left(\lambda \backslash\left(\lambda^{(1)}, \ldots, \lambda^{(k)}\right)\right)=\emptyset .
$$

In this way, we form a tree of partitions of $0, w_{1}, \ldots, w_{k}$ and corresponding to each branch of the tree is a separation of $\lambda$. For example, the separation of the partition $\lambda=\left(1^{7} 3^{2} 5\right)$ corresponding to the composition $w=(7,5,6)$ is represented by the following tree.

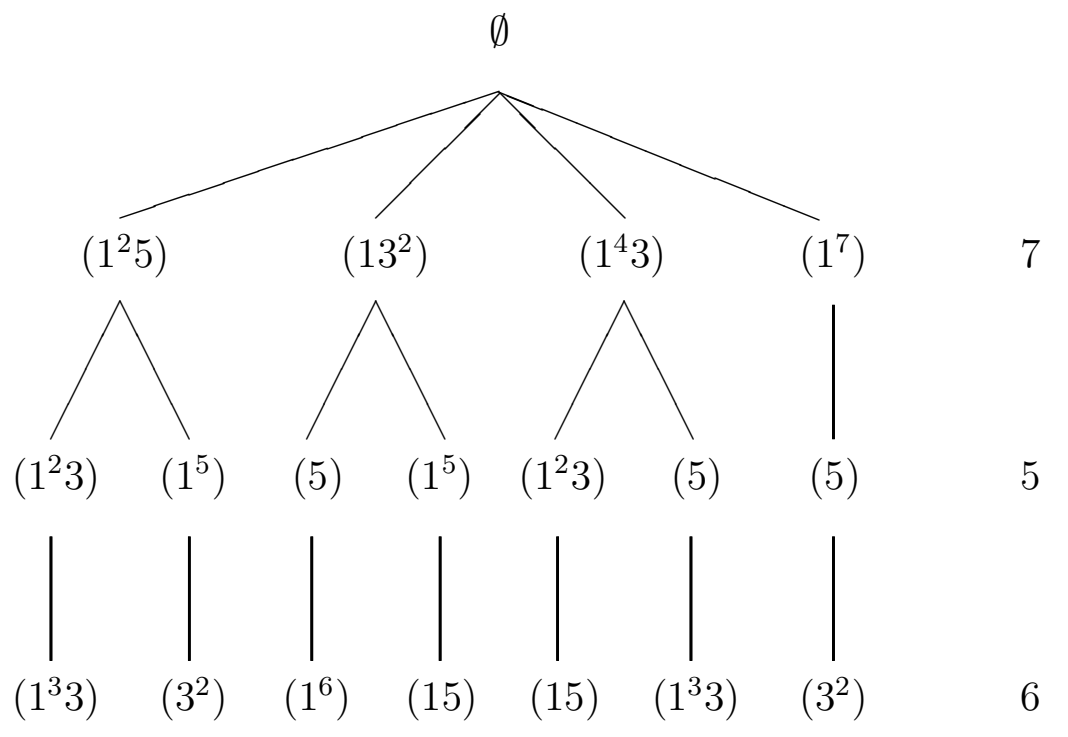




\subsection{Hall-Littlewood Symmetric Functions}

Let $\mathbf{x}=\left\{x_{1}, x_{2}, \ldots, x_{\ell}\right\}$ be a set of $\ell$ independent indeterminates and $t$ an independent parameter. If $\alpha=\left(\alpha_{1}, \alpha_{2}, \ldots, \alpha_{\ell}\right) \in \mathbf{Z}^{\ell}$, write $e^{\alpha}:=x_{1}^{\alpha_{1}} \ldots x_{\ell}^{\alpha_{\ell}}$ and

$$
\theta_{\ell}(\mathbf{x}, t)=\prod_{1 \leq i<j \leq \ell} \frac{x_{i}-t x_{j}}{x_{i}-x_{j}} .
$$

If $\lambda$ is a partition with $\ell(\lambda)=m \leq \ell$, put $\lambda_{i}=0$ for $i=m+1, \ldots, \ell$. Then the Hall-Littlewood symmetric functions $P_{\lambda}(\mathbf{x} ; t)$ are defined by

$$
P_{\lambda}(\mathbf{x} ; t)=\nu_{\lambda}(t)^{-1} \sum_{w \in S_{\ell}} w\left(e^{\lambda} \theta_{\ell}(\mathbf{x} ; t)\right)
$$

where $S_{\ell}$ is the symmetric group acting on $\mathbf{x}$,

$$
\nu_{\lambda}(t)=\prod_{i \geq 0} \nu_{n_{i}}(t), \quad \nu_{r}(t)=(1-t)^{-r} \prod_{i=1}^{r}\left(1-t^{j}\right)
$$

and $n_{i}:=n_{i}(\lambda)$ is the multiplicity of $i$ in $\lambda$. Alternatively, as shown by Macdonald [4]

$$
P_{\lambda}(\mathbf{x} ; t)=\sum_{w \in S_{\ell} / S_{\ell}^{\lambda}} w\left(e^{\lambda} \theta_{\ell}(\mathbf{x} ; t)\right)
$$

where $S_{\ell}^{\lambda}$ is the subgroup of $w \in S_{\ell}$ such that $\lambda_{w(i)}=\lambda_{i}(1 \leq i \leq \ell)$, and the summation is taken over left coset representatives of $S_{\ell}^{\lambda}$ in $S_{\ell}$. In fact, $S_{\ell}^{\lambda}=\prod_{i \geq 0} S_{n_{i}}$. We are going to be more concerned with another version of the Hall-Littlewood symmetric functions, namely

$$
Q_{\lambda}(\mathbf{x} ; t):=b_{\lambda}(t) P_{\lambda}(\mathbf{x} ; t)
$$

where $b_{\lambda}(t):=\prod_{i \geq 1} \prod_{j=1}^{n_{i}}\left(1-t^{j}\right)$. In particular, we put $q_{k}(\mathbf{x} ; t)=Q_{(k)}(\mathbf{x} ; t)$ and note that from (2.1) we have

$$
q_{k}(\mathbf{x} ; t)=(1-t) \sum_{i=1}^{\ell} x_{i}^{k} \beta_{i}(\mathbf{x} ; t)
$$

where

$$
\beta_{i}(\mathbf{x} ; t)=\prod_{\substack{j=1 \\ i \neq j}} \frac{x_{i}-t x_{j}}{x_{i}-x_{j}} \quad(i=1,2, \ldots, \ell) .
$$

Macdonald [4] has given the following useful recurrence relation for the $Q_{\lambda}(\mathbf{x} ; t)$.

$$
Q_{\lambda}(\mathbf{x} ; t)=(1-t) \sum_{i=1}^{\ell} x_{i}^{\lambda_{1}} \beta_{i}(\mathbf{x} ; t) Q_{\lambda \backslash\left\{\lambda_{1}\right\}}\left(\mathbf{x} \backslash\left\{x_{i}\right\} ; t\right)
$$

We are, in particular, interested in the case corresponding to $t=-1$, as these are the so-called Schur $Q$-functions which were introduced by I. Schur [6] to deal with the spin characters of 
symmetric groups. In this case, we have

$$
\left.\begin{array}{l}
\text { (i) If } \lambda \in P(\ell) \text {, then } b_{\lambda}(-1)=\left\{\begin{array}{cc}
2^{\ell(\lambda)} & \text { if } \lambda \in S P(\ell), \\
0 & \text { otherwise, }
\end{array}\right. \\
\text { (ii) } Q_{\lambda}(\mathbf{x} ;-1)=\left\{\begin{array}{r}
2^{\ell(\lambda)} P_{\lambda}(\mathbf{x} ;-1) \text { if } \lambda \in S P(\ell), \\
0 \text { otherwise, }
\end{array}\right. \\
\text { (iii) } q_{k}(\mathbf{x} ;-1)=2 \sum_{i=1}^{\ell} x_{i}^{k} \beta_{i}(\mathbf{x} ;-1) \quad(k=1,2, \ldots) .
\end{array}\right\}
$$

From now on, unless otherwise stated, we assume that $\lambda \in S P(\ell)$. Furthermore, in order to simplify the notation, we write $Q_{\lambda}(\mathbf{x})$ for $Q_{\lambda}(\mathbf{x},-1), q_{k}(\mathbf{x})$ for $q_{k}(\mathbf{x},-1), \beta_{k}(\mathbf{x})$ for $\beta_{k}(\mathbf{x},-1), \theta_{\ell}(\mathbf{x})$ for $\theta_{\ell}(\mathbf{x},-1)$, etc.

I. Schur [6] defines $Q$-functions by a recursive formula and then determines a closed formula similar to (2.2) above. We reverse the process and show that the $Q_{\lambda}(\mathbf{x})$ as defined above satisfy certain properties, including the recurrence properties used in I. Schur's definition.

If $\lambda=\left(\lambda_{1}, \ldots, \lambda_{m}\right) \in S P(\ell)$, then from (2.2) and (2.6)(ii), we have

$$
Q_{\lambda}(\mathbf{x})=2^{m} \sum_{w \in S_{\ell} / S_{\ell-m}} w\left(e^{\lambda} \theta_{\ell}(\mathbf{x})\right)
$$

where $S_{\ell-m}$ is the subgroup of $S_{\ell}$ which fixes $x_{m+1}, \ldots, x_{\ell}$ and the summation is over all left coset representatives of $S_{\ell-m}$ in $S_{\ell}$. Thus, we have

$$
Q_{\lambda}(\mathbf{x})=2^{m} \sum_{w \in S_{\ell} / S_{\ell-m}}(-1)^{\frac{m(m-1)}{2}} w\left(e^{\lambda} \frac{\theta_{m}(\mathbf{x})}{\prod_{i=1}^{m} \beta_{i}(\mathbf{x})}\right),
$$

which is the form of the $Q$-function obtained by I. Schur.

We now proceed to show that the recurrence relations used to define Schur's $Q$-functions are satisfied. In order to do this, we need the following which was indeed proved by I. Schur [6, p.226].

$$
\theta_{m}(\mathbf{x})=\sum_{i=2}^{m}(-1)^{i} \theta_{2}\left(x_{1}, x_{i}\right) \theta_{m-2}\left(\mathbf{x} \backslash\left\{x_{1}, x_{i}\right\}\right) \quad \text { if } m \text { is even }
$$

and

$$
\theta_{m}(\mathbf{x})=\sum_{i=1}^{m}(-1)^{i-1} \theta_{m-1}\left(\mathbf{x} \backslash\left\{x_{i}\right\}\right) \text { if } \quad m \text { is odd. }
$$

Theorem 2.2 Let $\lambda=\left(\lambda_{1}, \ldots, \lambda_{m}\right) \in S P(\ell)$. Then

(i) $Q_{(r, s)}(\mathbf{x})=q_{r}(\mathbf{x}) q_{s}(\mathbf{x})+2 \sum_{i=1}^{s}(-1)^{s} q_{r+i}(\mathbf{x}) q_{s-i}(\mathbf{x})$.

(ii) If $m$ is even

$$
Q_{\lambda}(\mathbf{x})=\sum_{i=2}^{m}(-1)^{i} Q_{\left(\lambda_{1}, \lambda_{i}\right)}(\mathbf{x}) Q_{\lambda \backslash\left\{\lambda_{1}, \lambda_{i}\right\}},(\mathbf{x}) .
$$

(iii) If $m$ is odd

$$
Q_{\lambda}(\mathbf{x})=\sum_{i=1}^{m}(-1)^{i-1} q_{\lambda_{i}}(\mathbf{x}) Q_{\lambda \backslash\left\{\lambda_{i}\right\}}(\mathbf{x})
$$


Proof (i) From (2.5) we have

$$
\begin{aligned}
Q_{(r, s)}(\mathbf{x}) & =\sum_{i=1}^{\ell} x_{i}^{r} \beta_{i}(\mathbf{x}) q_{s}\left(\mathbf{x} \backslash\left\{x_{i}\right\}\right) \\
& =\sum_{i=1}^{\ell} x_{i}^{r} \beta_{i}(\mathbf{x})\left(\sum_{j=1}^{\ell} x_{j}^{s} \beta_{j}(\mathbf{x}) \frac{x_{j}-x_{i}}{x_{j}+x_{i}}\right) \\
& =\sum_{i=1}^{\ell} x_{i}^{r} \beta_{i}(\mathbf{x})\left(\sum_{j=1}^{\ell} x_{j}^{s} \beta_{j}(\mathbf{x})\left(1-\frac{2 x_{i}}{x_{j}+x_{i}}\right)\right),
\end{aligned}
$$

from which we deduce that

$$
Q_{(r, s)}(\mathbf{x})=q_{r}(\mathbf{x}) q_{s}(\mathbf{x})-2 \sum_{i=1}^{\ell} x_{i}^{r+1} \beta_{i}(\mathbf{x})\left(\sum_{j=1}^{\ell} x_{j}^{s} \beta_{j}(\mathbf{x}) \frac{1}{x_{j}+x_{i}}\right) .
$$

A similar calculation, but now writing $\frac{x_{j}-x_{i}}{x_{j}+x_{i}}=-1+\frac{2 x_{j}}{x_{j}+x_{i}}$ gives

$$
Q_{(r+1, s-1)}(\mathbf{x})=-q_{r+1}(\mathbf{x}) q_{s-1}(\mathbf{x})+2 \sum_{i=1}^{\ell} x_{i}^{r-1} \beta_{i}(\mathbf{x})\left(\sum_{j=1}^{\ell} x_{j}^{s} \beta_{j}(\mathbf{x}) \frac{1}{x_{j}+x_{i}}\right) .
$$

Now, adding (2.11) and (2.12), we have

$$
Q_{(r+1, s-1)}(\mathbf{x})=q_{r}(\mathbf{x}) q_{s}(\mathbf{x})-q_{r+1}(\mathbf{x}) q_{s-1}(\mathbf{x})-Q_{(r, s)}(\mathbf{x}) .
$$

A similar calculation to the above shows that

$$
Q_{(r, 1)}(\mathbf{x})=q_{r}(\mathbf{x}) q_{1}(\mathbf{x})-2 q_{r+1}(\mathbf{x})
$$

and the proof is now completed by induction on $s$.

(ii) If $m$ is even, by substituting (2.9) in (2.8), we obtain

$$
\begin{aligned}
Q_{\lambda}(\mathbf{x})= & 2^{m} \sum_{w \in S_{\ell} / S_{\ell-m}}(-1)^{m(m-1) / 2} w\left(\frac{e^{\lambda} \sum_{i=2}^{m}(-1)^{i} \theta_{2}\left(x_{1}, x_{i}\right) \theta_{m-2}\left(\mathbf{x} \backslash\left\{x_{1}, x_{i}\right\}\right)}{\prod_{j=1}^{m} \beta_{j}(\mathbf{x})}\right) \\
= & \sum_{i=2}^{m}(-1)^{i}\left(2^{2} \sum_{w \in S_{\ell} / S_{\ell-2}}-w\left(e^{\left(\lambda_{1}, \lambda_{i}\right)} \frac{\theta_{2}\left(x_{1}, x_{i}\right)}{\beta_{1}(\mathbf{x}) \beta_{i}(\mathbf{x})}\right)\right) \\
& \left(2^{m-2} \sum_{w \in S_{\ell} / S_{\ell-m+2}}(-1)^{(m-2)(m-3) / 2} w\left(\frac{\left(e^{\left(\lambda \backslash\left\{\lambda_{1}, \lambda_{i}\right\}\right)} \theta_{m-2}\left(\mathbf{x} \backslash\left\{x_{1}, x_{i}\right\}\right)\right.}{\prod_{\substack{j=2 \\
j \neq i}}^{m} \beta_{j}(\mathbf{x})}\right)\right) \\
= & \sum_{i=2}^{m}(-1)^{i} Q_{\left(\lambda_{1}, \lambda_{i}\right)}(\mathbf{x}) Q_{\lambda \backslash\left\{\lambda_{1}, \lambda_{i}\right\}}(\mathbf{x})
\end{aligned}
$$

The proof of (iii) is similar, but using (2.10).

Macdonald [4, p.109] has shown that the inductive formula (2.5) can be used to extend the definition of the Schur $Q$-functions to any $Q_{\mu}(\mathbf{x})$, where $\mu=\left(\mu_{1}, \ldots, \mu_{k}\right) \in \mathbf{Z}^{k}$, for any $k$. Furthermore, Theorem 2.1(i) has been used to reduce such a $Q_{\mu}(\mathbf{x})$ to a linear combination of $Q_{\lambda}(\mathbf{x})$ such that $\lambda \in S P(\ell)$ (see also Morris [5]). The reduction rules are as follows. 
Theorem 2.3 (i) $Q_{(\ldots, r, s, \ldots)}(\mathbf{x})=--Q_{(\ldots, s, r, \ldots)}(\mathbf{x})$ with $Q_{(\ldots, i, \ldots, i, \ldots)}(\mathbf{x})=0$.

(ii) $Q_{\ldots, r,-r, \ldots)}(\mathbf{x})=0, Q_{\left(\mu_{1}, \ldots, \mu_{i-1},-r, r, \mu_{i+1}, \ldots, \mu_{n}\right)}(\mathbf{x})=2(-1)^{r} Q_{\left(\mu_{1}, \ldots, \mu_{i-1}, \mu_{i+1}, \ldots, \mu_{n}\right)}(\mathbf{x})$;

in particular $Q_{(s, r)}(\mathbf{x})=-Q_{(r, s)}(\mathbf{x}), \quad r \neq s$ and $Q_{(-r, r)}(\mathbf{x})=2(-1)^{r}$.

A consequence of (i) is that if $\lambda=\left(\lambda_{1}, \ldots, \lambda_{m}\right) \in S P(\ell)$, then

$$
Q_{\lambda}(\mathbf{x})=\epsilon(\sigma) Q_{\left(\lambda_{\sigma(1)}, \ldots, \lambda_{\sigma(m)}\right)}
$$

for all $\sigma \in S_{m}$, where $\epsilon(\sigma)$ is the sign of the permutation $\sigma$.

\section{$3 \quad$ Further Results on Schur $Q$-functions}

¿From now on, it will be essential that it is clear on what sets our symmetric groups act, thus the notation will be modified. We denote by $S_{\underline{m}}$ the symmetric group which acts on the set $\underline{m}=\{1,2, \ldots, m\}$ and $S_{\underline{m} \backslash k}$ the symmetric group which acts on $\underline{m} \backslash \underline{k}=\{k+1, \ldots, m\}$. Also, we need to determine certain explicit left coset representations of certain groups. These are given by the following.

Lemma 3.1 For $k=2, \ldots, m$, put $w_{k}=(2 k) \in S_{\underline{m}}$ and for $k=1,2, \ldots, m$, put $u_{k}=(1 k) \in$ $S_{\underline{m}}$. Then

(i) $\left\{w_{k} \mid k=2, \ldots, m\right\}$ is a set of left coset representatives of $S_{\underline{m} \backslash \underline{2}}$ in $S_{\underline{m} \backslash \underline{1}}$.

(ii) $\left\{u_{k} \mid k=1, \ldots, m\right\}$ is a set of left coset representatives of $S_{\underline{m} \backslash \underline{1}}$ in $S_{\underline{m}}$.

We can now use the following lemma to give an alternative form for the $Q_{\lambda}(\mathbf{x})$.

Lemma 3.2 If $\lambda=\left(\lambda_{1}, \ldots, \lambda_{m}\right) \in S P(\ell)$, then

$$
Q_{\lambda}(\mathbf{x})=\sum_{\eta \in S_{\underline{m} \backslash \underline{1}} / S_{\underline{m} \backslash \underline{2}}} \epsilon(\eta) Q_{\left(\lambda_{1}, \lambda_{\eta(2)}\right)}(\mathbf{x}) Q_{\left(\lambda_{\eta(3)}, \ldots, \lambda_{\eta(m)}\right)}(\mathbf{x})
$$

if $m$ is even, and

$$
Q_{\lambda}(\mathbf{x})=\sum_{\eta \in S_{\underline{m}} / S_{\underline{m} \backslash \underline{1}}} \epsilon(\eta) q_{\eta(1)}(\mathbf{x}) Q_{\left(\lambda_{\eta(2)}, \ldots, \lambda_{\eta(m)}\right)}(\mathbf{x})
$$

if $m$ is odd.

Proof If $\eta \in S_{\underline{m} \backslash \underline{1}}$, then by Lemma 3.1(i) we have $\eta=w_{k} \nu_{k}$, where $w_{k}=(2 k), \nu_{k} \in S_{\underline{m} \backslash \underline{2}}$ for some $2 \leq k \leq m$, and so $\eta=$

$$
\begin{aligned}
& (2 k)\left(\begin{array}{ccccccc}
3 & \ldots & k & \ldots & s & \ldots & m \\
\eta(3) & \ldots & \eta(k) & \ldots & \eta(2) & \ldots & \eta(m)
\end{array}\right)=\left(\begin{array}{cccccccc}
2 & 3 & \ldots & k & \ldots & s & \ldots & m \\
k & \eta(3) & \ldots & \eta(k) & \ldots & 2 & \ldots & \eta(m)
\end{array}\right) \\
& =(k, \eta(k))\left(\begin{array}{cccccccc}
2 & 3 & \ldots & k & \ldots & s & \ldots & m \\
\eta(k) & \eta(3) & \ldots & \eta(2) & \ldots & \eta(s) & \ldots & \eta(m)
\end{array}\right) \text {, }
\end{aligned}
$$


where $\eta(2)=k$ and $\eta(s)=2$. Hence, if $m$ is even

$$
\begin{aligned}
& \sum_{\eta \in S_{\underline{m} \backslash \underline{1}} / S_{\underline{m} \backslash \underline{2}}} \epsilon(\eta) Q_{\left(\lambda_{1}, \lambda_{\eta(n)}\right)}(\mathbf{x}) Q_{\left(\lambda_{\eta(3)}, \ldots, \lambda_{\eta(m)}\right)}(\mathbf{x}) \\
& =\sum_{k=2}^{m} \epsilon(k, \eta(k)) \epsilon\left(\begin{array}{cccccccc}
2 & 3 & \ldots & k & \ldots & s & \ldots & m \\
\eta(k) & \eta(3) & \ldots & \eta(2) & \ldots & \eta(s) & \ldots & \eta(m)
\end{array}\right) Q_{\left(\lambda_{1}, \lambda_{k}\right)}(\mathbf{x}) Q_{\left(\lambda_{\eta(3)}, \ldots, \lambda_{\eta(m)}\right)}(\mathbf{x}) \\
& =\sum_{k=2}^{m} \epsilon(k, \eta(k)) Q_{\left(\lambda_{1}, \lambda_{k}\right)}(\mathbf{x}) Q_{\left(\lambda_{3}, \ldots, \lambda_{2}, \ldots, \lambda_{m}\right)}(\mathbf{x})
\end{aligned}
$$

with $\lambda_{2}$ in the $k$ position in $Q_{\left(\lambda_{3}, \ldots, \lambda_{2}, \ldots, \lambda_{m}\right)}(\mathbf{x})$, where we have used (2.13). Now, by using Theorem 2.3(i) repeatedly, we have that the right hand side of the above reduces to

$$
\sum_{k=2}^{m}(-1)^{k-2} Q_{\left(\lambda_{1}, \lambda_{k}\right)}(\mathbf{x}) Q_{\lambda \backslash\left\{\lambda_{1}, \lambda_{k}\right\}}(\mathbf{x})=Q_{\lambda}(\mathbf{x})
$$

by Theorem 2.2(ii). A similar argument can be used to prove (ii).

Lemma 3.3 If $m$ is even and $\lambda \in S P(\ell)$, then

$$
Q_{\lambda}(\mathbf{x})=\frac{1}{m} \sum_{\sigma \in S_{\underline{m}} / S_{\underline{m} \backslash \underline{2}}} \epsilon(\sigma) Q_{\left(\lambda_{\sigma(1)}, \lambda_{\sigma(2)}\right)}(\mathbf{x}) Q_{\left(\lambda_{\sigma(3)}, \ldots, \lambda_{\sigma(m)}\right)}(\mathbf{x})
$$

Proof If $m$ is even, then from (2.13), Theorem 2.2(ii) and Lemma 3.2, we have

$$
\begin{aligned}
Q_{\lambda}(\mathbf{x}) & =\frac{1}{m} \sum_{\sigma \in S_{\underline{m}} / S_{\underline{m} \backslash \underline{1}}} \epsilon(\sigma) Q_{\left(\lambda_{\sigma(1)}, \ldots, \lambda_{\sigma(m)}\right)}(\mathbf{x}) \\
& =\frac{1}{m} \sum_{\sigma \in S_{\underline{m}} / S_{\underline{m} \backslash \underline{1}}} \epsilon(\sigma) \sum_{i=2}^{m}(-1)^{i} Q_{\left(\lambda_{\sigma(1)}, \lambda_{\sigma(i)}\right)}(\mathbf{x}) Q_{\left(\lambda_{\sigma(2)}, \ldots, \lambda_{\sigma(m)}\right) \backslash\left\{\lambda_{\sigma(1)}, \lambda_{\sigma(i)}\right\}}(\mathbf{x}) \\
& =\frac{1}{m} \sum_{\sigma \in S_{\underline{m}} / S_{\underline{m} \backslash \underline{1}}} \epsilon(\sigma) \sum_{\eta \in S_{\underline{m} \backslash \underline{1}} / S_{\underline{m} \backslash \underline{2}}} \epsilon(\eta) Q_{\left(\lambda_{\sigma \eta(1)}, \lambda_{\sigma \eta(2)}\right)}(\mathbf{x}) Q_{\left(\lambda_{\sigma \eta(3)}, \ldots, \lambda_{\sigma \eta(m)}\right)}(\mathbf{x}) \\
& =\frac{1}{m} \sum_{\sigma \in S_{\underline{m}} / S_{\underline{m} \backslash \underline{2}}} \epsilon(\sigma) Q_{\left(\lambda_{\sigma(1)}, \lambda_{\sigma(2)}\right)}(\mathbf{x}) Q_{\left(\lambda_{\sigma(3)}, \ldots, \lambda_{\sigma(n)}\right)}(\mathbf{x}) .
\end{aligned}
$$

We can now use this result as the basis for an inductive proof of the following.

Theorem 3.4 (i) If $m=2 \mu$ is even, then

$$
Q_{\lambda}(\mathbf{x})=\frac{1}{2^{\mu} \mu !} \sum_{\sigma \in S_{\underline{m}}} \epsilon(\sigma) Q_{\left(\lambda_{\sigma(1)}, \lambda_{\sigma(2)}\right)}(\mathbf{x}) Q_{\left(\lambda_{\sigma(3)}, \lambda_{\sigma(4)}\right)}(\mathbf{x}) \ldots Q_{\left(\lambda_{\sigma(m-1)}, \lambda_{\sigma(m)}\right)}(\mathbf{x})
$$

(ii) If $m=2 \mu+1$ is odd, then

$$
Q_{\lambda}(\mathbf{x})=\frac{1}{2^{\mu} \mu !} \sum_{\sigma \in S_{\underline{m}}} \epsilon(\sigma) q_{\lambda_{\sigma(1)}}(\mathbf{x}) Q_{\left(\lambda_{\sigma(2)}, \lambda_{\sigma(3)}\right)}(\mathbf{x}) Q_{\left(\lambda_{\sigma(4)}, \lambda_{\sigma(5)}\right)}(\mathbf{x}) \ldots Q_{\left(\lambda_{\sigma(m-1)}, \lambda_{\sigma(m)}\right)}(\mathbf{x})
$$


Proof (i) The proof is by induction on $m$. If $m=1$, then clearly

$$
Q_{\left(\lambda_{1}, \lambda_{2}\right)}(\mathbf{x})=\frac{1}{2} \sum_{\sigma \in S_{2}} \epsilon(\sigma) Q_{\left(\lambda_{\sigma(1)}, \lambda_{\sigma(2)}\right)}(\mathbf{x}) .
$$

If $m>1$, then by Lemma 3.3 and the inductive assumption, we have

$$
\begin{aligned}
& Q_{\lambda}(\mathbf{x})=\frac{1}{2 \mu} \sum_{\sigma \in S_{\underline{m}} / S_{\underline{m} \backslash \underline{2}}} \epsilon(\sigma) Q_{\left(\lambda_{\sigma(1)}, \lambda_{\sigma(2)}\right.}(\mathbf{x}) \frac{1}{2^{\mu-1}(\mu-1) !} \sum_{\rho \in S_{(\underline{m}) \backslash \sigma(2)}} \epsilon(\rho) Q_{\left(\lambda_{\rho \sigma(3)}, \lambda_{\rho \sigma(4)}\right)}(\mathbf{x}) \\
& \quad \ldots Q_{\left(\lambda_{\rho \sigma(m-1)}, \lambda_{\rho \sigma(m)}\right.}(\mathbf{x}) \\
&=\frac{1}{2^{\mu} \mu !} \sum_{\sigma \in S_{\underline{m}} / S_{\underline{m} \backslash \underline{2}}} \epsilon(\sigma) Q_{\left(\lambda_{\sigma(1)}, \lambda_{\sigma(2)}\right)}(\mathbf{x}) \sum_{\sigma^{\prime} \in S_{\underline{m} \backslash 2}} \epsilon\left(\sigma^{\prime}\right) Q_{\left.\lambda_{\sigma^{\prime}(3)}, \lambda_{\sigma^{\prime}(4)}\right)}(\mathbf{x}) \\
& \quad \ldots Q_{\lambda_{\left(\sigma^{\prime}(m-1)\right.}, \lambda_{\sigma^{\prime}(m)}}(\mathbf{x}) \\
&=\frac{1}{2^{\mu} \mu !} \sum_{\sigma \in S_{m}} \epsilon(\sigma) Q_{\left(\lambda_{\sigma(1)}, \lambda_{\sigma(2)}\right)}(\mathbf{x}) Q_{\left(\lambda_{\sigma(3)}, \lambda_{\sigma(4)}\right)}(\mathbf{x}) \ldots Q_{\left(\lambda_{\sigma(m-1)}, \lambda_{\sigma(m)}\right)}(\mathbf{x})
\end{aligned}
$$

as required. The proof of (ii) is similar.

The above result can be interpreted in terms of wreath products of certain groups; in fact, the hyperoctahedral group.

If $\lambda=\left(\lambda_{1}, \ldots, \lambda_{m}\right)$ where $m=2 \mu$ is even, let

$$
M=\left\{\left\{\lambda_{1}, \lambda_{2}\right\},\left\{\lambda_{3}, \lambda_{4}\right\}, \ldots,\left\{\lambda_{m-1}, \lambda_{m}\right\}\right\}
$$

and if $\sigma \in S_{m}$, let

$$
\sigma(M)=\left\{\left\{\lambda_{\sigma(1)}, \lambda_{\sigma(2)}\right\},\left\{\lambda_{\sigma(3)}, \lambda_{\sigma(4)}\right\}, \ldots,\left\{\lambda_{\sigma(m-1)}, \lambda_{\sigma(m)}\right\}\right\}
$$

If $H=\left\{\sigma \in S_{m} \mid \sigma(M)=M\right\}$, then $H$ is a subgroup of $S_{m}$ of order $2^{\mu} \mu$ !. In fact, if $\pi=$ $(12)(34) \ldots(m-1, m) \in S_{m}$, then $H$ is the centralizer $C_{S_{m}}(\pi)$ of $\pi$ in $S_{m}$, which implies that $H \cong S_{2} 2 S_{m}$ (see [2,p.135]), the wreath product of $S_{2}$ by $S_{m}$, which is the hyperoctahedral group or the Weyl group of type $B_{\mu}$. Thus, the above results can be rewritten as

Theorem 3.5 For $m \geq 1$, we have

(i) if $m=2 \mu$ is even

$$
Q_{\lambda}(\mathbf{x})=\sum_{\sigma \in S_{m} \backslash S_{2} \backslash S_{\mu}} \epsilon(\sigma) Q_{\left(\lambda_{\sigma(1)}, \lambda_{\sigma(2)}\right)}(\mathbf{x}) \ldots Q_{\left(\lambda_{\sigma(m-1)}, \lambda_{\sigma(m)}\right)}(\mathbf{x})
$$

(ii) if $m=2 \mu+1$ is odd

$$
Q_{\lambda}(\mathbf{x})=\sum_{\sigma \in S_{m} \backslash S_{2} l S_{\mu}} \epsilon(\sigma) q_{\lambda_{\sigma(1)}} Q_{\left(\lambda_{\sigma(2)}, \lambda_{\sigma(3)}\right)}(\mathbf{x}) \ldots Q_{\left(\lambda_{\sigma(m-1)}, \lambda_{\sigma(m)}\right)}(\mathbf{x})
$$




\section{Application to the Spin Characters of Symmetric Groups}

As was mentioned earlier, Schur $Q$-functions were first introduced by Schur [6] in his work on the spin (projective) characters of symmetric groups (see also [1]). He showed that the irreducible spin characters of $S_{\ell}$ were parameterised by strict partition $\lambda \in S P(\ell)$, but that this correspondence is not one-to-one. In fact, if we say that $\lambda$ is even (odd) if $\ell-\ell(\lambda)$ is even (odd), there is one irreducible self-associate spin character $\zeta^{\lambda}$ corresponding to $\lambda \in S P(n)$ if $\lambda$ is even and two irreducible associate spin characters $\zeta^{\lambda}$ and $\zeta^{\lambda^{\prime}}$ if $\lambda$ is odd. The connection with Schur $Q$-functions is given by

$$
Q_{\lambda}(\mathbf{x})=\sum_{\pi \in O(\ell)} 2^{\frac{1}{2}(\ell(\lambda)+\ell(\pi)+\epsilon(\lambda))} z_{\pi}^{-1} \zeta_{\pi}^{\lambda} p_{\pi}(\mathbf{x})
$$

where $z_{\pi}=\prod_{i \geq 1} i^{m_{i}} m_{i}$ !, where $m_{i}$ is the multiplicity of $i$ in the partition $\pi=\left(1^{m_{1}} 3^{m_{3}} \ldots\right)$, $p_{\pi}(\mathbf{x})=p_{1}(\mathbf{x})^{m_{1}} p_{3}(\mathbf{x})^{m_{3}} \ldots\left(p_{i}(\mathbf{x})=\sum_{k=1}^{\ell} x_{k}^{i}\right), \zeta_{\pi}^{\lambda}$ is the value of the character $\zeta^{\lambda}$ at the class $\pi$ and

$$
\epsilon(\lambda)= \begin{cases}0 & \text { if } \ell-\ell(\lambda) \text { is even } \\ 1 & \text { if } \ell-\ell(\lambda) \text { is odd }\end{cases}
$$

This formula gives the value of $\zeta^{\lambda}$ on the classes $\pi \in O(\ell)$ only; however, if $\lambda$ is even $\zeta_{\pi}^{\lambda}=0$ if $\pi \in P(\ell) \backslash O(\ell)$ and if $\lambda$ is odd $\zeta_{\pi}^{\lambda}=\zeta_{\pi}^{\lambda^{\prime}}=\zeta_{\pi}^{\lambda^{\prime}}$ if $\pi \in O(\ell)$, while if $\pi \notin O(\ell), \zeta_{\pi}^{\lambda} \neq 0$ only if $\pi=\lambda$ and then $\zeta_{\lambda}^{\lambda}$ is given explicitly by

$$
\zeta_{\lambda}^{\lambda}=(-1)^{(-\ell(\lambda)+1)} \sqrt{\frac{\lambda_{1} \lambda_{2} \ldots \lambda_{\ell(\lambda)}}{2}}
$$

and $\zeta_{\lambda}^{\lambda^{\prime}}=-\zeta_{\lambda}^{\lambda}$. Thus, formula (4.16) gives all the information required. In addition, we require

$$
\zeta_{\pi}^{(\ell)}=2^{\frac{1}{2}(\ell(\sigma)-1-\epsilon)}
$$

where

$$
\epsilon= \begin{cases}1 & \text { if } \ell \text { is odd } \\ 0 & \text { if } \ell \text { is even }\end{cases}
$$

and

$$
q_{\ell}(\mathbf{x})=\sum_{\pi \in O(\ell)} 2^{\ell(\pi)} z_{\pi}^{-1} p_{\pi}(\mathbf{x})
$$

Both results are originally due to Schur [6]; in fact (4.19) is (4.16) for the particular case $\lambda=(\ell)$ using the value given by (4.18) for the character $\zeta_{\pi}^{(\ell)}$.

Our intention now is to give a recursive formula for the calculation of the $\zeta_{\pi}^{\lambda}$ based on the preceding work on Schur $Q$-functions.

We shall assume from now on that $\pi=\left(1^{m_{1}} 3^{m_{3}} \ldots\right) \in O(\ell)$. Let $\left\{\pi^{1}, \pi^{2}, \ldots, \pi^{m}\right\}$ be a separation of $\pi$; then we note that

$$
\frac{z_{\pi}}{z_{\pi^{1}} z_{\pi^{2}} \ldots z_{\pi^{m}}}=\frac{y_{\pi}}{y_{\pi^{1}} y_{\pi^{2}} \ldots y_{\pi^{m}}}
$$

where $y_{\pi}=\prod_{i \geq 1} m_{i}$ ! (recall that $\left.z_{\pi}=\prod_{i \geq 1} i^{m_{i}} m_{i} !\right)$.

We first give an explicit formula for $\zeta_{\pi}(r, s)(r>s)$, that is for two part partitions. We prove 
Theorem 4.1 If $\lambda=(r, s) \in S P(\ell)(r>s), \pi \in O(\ell)$, then

$$
\zeta_{\pi}^{(r, s)}=2^{\frac{1}{2}(\ell(\pi)-2-\epsilon)} y_{\pi}\left\{\sum_{\left(\pi_{r}, \pi_{s}\right)=\pi} y_{\pi_{r}}^{-1} y_{\pi_{s}}^{-1}+2 \sum_{j=1}^{s}(-1)^{j}\left(\sum_{\left(\pi_{r+j}, \pi_{s-j}\right)} y_{r+j}^{-1} y_{s-j}^{-1}\right)\right\}
$$

where the sums are taken over all separations $\left(\pi_{r+j}, \pi_{s-j}\right)$ of $\pi$ corresponding to the composition (partition) $(r+j, s-j)$ and $\epsilon=0(1)$ if $\ell$ is even (odd).

Proof For the partition $(r, s) \in S P(\ell)$, (4.16) becomes

$$
Q_{(r, s)}(\mathbf{x})=\sum_{\pi \in O(\ell)} 2^{\frac{1}{2}(\ell(\pi)+2+\epsilon)} z_{\pi}^{-1} \zeta_{\pi}^{(r, s)} p_{\pi}(\mathbf{x})
$$

where $\epsilon=0(1)$ if $\ell$ is even (odd). Now, by substituting (4.19) and (4.21) in Theorem 2.2(i), we obtain

$$
\begin{array}{r}
\sum_{\pi \in O(\ell)} 2^{\frac{1}{2}(\ell(\pi)+2+\epsilon)} z_{\pi}^{-1} \zeta_{\pi}^{(r, s)} p_{\pi}(\mathbf{x})=\left(\sum_{\pi_{r} \in O(r)} z_{\pi_{r}}^{-1} 2^{\ell\left(\pi_{r}\right)} p_{\pi_{r}}(\mathbf{x})\right)\left(\sum_{\pi_{s} \in O(s)} z_{\pi_{s}}^{-1} 2^{\ell\left(\pi_{s}\right)} p_{\pi_{s}(\mathbf{x})}\right) \\
+2 \sum_{j=1}^{s}(-1)^{j}\left(\sum_{\pi_{r+j} \in O(r+j)} z_{\pi_{r+j}}^{-1} 2^{\ell\left(\pi_{r+j}\right)} p_{\pi_{r+j}}(\mathbf{x})\right) \\
=\sum_{\left(\pi_{r}, \pi_{s}\right)=\pi \in O(\ell)} z_{\pi_{r}}^{-1} z_{\pi_{s}}^{-1} 2^{\ell(\pi)} p_{\pi}(\mathbf{x})+2 \sum_{j=1}^{s}(-1)^{j} \sum_{\left(\pi_{r+j}, \pi_{s-j}\right)=\pi \in O(\ell)} z_{\pi_{r+j}}^{-1} z_{\pi_{s-j}}^{-1} 2^{\ell(\pi)} p_{\pi}(\mathbf{x}),
\end{array}
$$

where the sums are taken over all separations $\left(\pi_{r+j}, \pi_{s-j}\right)$ of $\pi$ corresponding to the composition $(r+j, s-j)$. Now, by comparing the coefficients of $p_{\pi}(\mathbf{x})$ on both sides of this equation and using (4.19), we obtain the required result.

Thus, the calculation of $\zeta_{\pi}^{(r, s)}$ for $\pi \in O(\ell)$, is reduced to a calculation involving the separation of partitions and is given in combinatorial terms.

Example: We calculate $\zeta_{\left(1^{6} 3\right)}^{(6,3)}$. The separations of $\left(1^{6} 3\right)$ corresponding to the composition $(6,3),(7,2),(8,1)$ and $(9)$ are $\left(\left\{\left(1^{6}\right),(3)\right\},\left\{\left(1^{3} 3\right),\left(1^{3}\right)\right\}\right),\left\{\left(1^{4} 3\right),\left(1^{2}\right)\right\},\left\{\left(1^{5} 3\right),(1)\right\}$ and $\left\{\left(1^{6} 3\right), \emptyset\right\}$ respectively. Thus

$$
\begin{aligned}
\chi_{\left(1^{6} 3\right)}^{(6,3)} & =2^{2} 6 !\left\{\left(\frac{1}{6 !}+\frac{1}{3 ! 3 !}\right)+2\left(-\frac{1}{4 ! 2 !}+\frac{1}{5 !}-\frac{1}{6 !}\right)\right\} \\
& =4 .
\end{aligned}
$$

We now use the expansion of the Schur $Q$-functions $Q_{\lambda}(\mathbf{x})$ in terms of products of Schur $Q$ functions corresponding to two-part partitions to give a combinatorial formula for $\zeta_{\pi}^{\lambda}$ in the general case. We use Theorem 3.5 (or Theorem 3.4). We prove

Theorem 4.2 (i) If $m=2 \mu$ is even and $\lambda=\left(\lambda_{1}, \ldots, \lambda_{m}\right) \in S P(\ell), \pi=\left(1^{m_{1}} 3^{m_{3}} \ldots\right) \in O(\ell)$, $I=\{1,3,5, \ldots, m-1\}$, then

$$
\zeta_{\pi}^{\lambda}=\sum_{\sigma \in S_{m} / S_{2} l S_{\mu}} \epsilon(\sigma)\left\{\sum_{\left(\pi_{1}^{\sigma}, \pi_{3}^{\sigma}, \ldots\right)=\pi} y_{\pi}\left(\prod_{i \in I} y_{\pi_{i}^{\sigma}}^{-1}\right) 2^{\frac{1}{2}\left(-\epsilon+\sum_{i \in I} \epsilon_{i}^{\sigma}\right)} \prod_{i \in I} \zeta_{\pi_{i}^{\sigma}}^{\left(\lambda_{\sigma(i)}, \lambda_{\sigma(i+1)}\right)}\right\},
$$


where for each $i \in I, \pi_{i}^{\sigma}=\pi_{\left(\lambda_{\sigma(i)}, \lambda_{\sigma(i+1)}\right)} \in O\left(\left|\lambda_{\sigma(i)}\right|+\left|\lambda_{\sigma(i+1)}\right|\right), \epsilon_{i}^{\sigma}=0(1)$ if $\left|\lambda_{\sigma(i)}\right|+\left|\lambda_{\sigma(i+1)}\right|$ is even (odd), $\epsilon=0(1)$ if $\ell$ is even (odd), and the sum is taken over all separations $\left(\pi_{1}^{\sigma}, \pi_{3}^{\sigma}, \ldots, \pi_{m-1}^{\sigma}\right)$ of $\pi$.

(ii) If $m=2 \mu+1$ is odd and $\lambda=\left(\lambda_{1}, \ldots, \lambda_{m}\right) \in S P(\ell), \pi=\left(1^{m_{1}} 3^{m_{3}} \ldots\right) \in O(\ell)$, $J=\{2,4, \ldots, m-1\}$, then

$$
\begin{gathered}
\zeta_{\pi}^{\lambda}=\sum_{\sigma \in S_{m} / S_{2} l S_{\mu}} \epsilon(\sigma)\left\{\sum_{\left(\pi_{\left.\lambda_{\sigma(1)}\right)}, \pi_{2}^{\sigma}, \pi_{4}^{\sigma}, \ldots, \pi_{m-1}^{\sigma}\right)=\pi} y_{\pi} y_{\pi_{\lambda_{\sigma(1)}}^{-1}} \prod_{j \in J} y_{\pi_{j}^{\sigma}}^{-1}\right. \\
\left.2^{\left.\frac{1}{2}\left(-\epsilon+\epsilon_{\sigma(1)}+\sum_{j \in J} \epsilon_{j}^{\sigma}\right)\right)} \zeta_{\pi_{\lambda_{\sigma(1)}}}^{\left(\lambda_{\sigma(1)}\right)} \prod_{j \in J} \zeta_{\pi_{j}^{\sigma}}^{\left(\lambda_{\sigma(j)}, \lambda_{\sigma(j+1)}\right)}\right),
\end{gathered}
$$

where for each $j \in J, \pi_{j}^{\sigma}=\pi_{\left(\lambda_{\sigma(j)}, \lambda_{\sigma(j+1)}\right)} \in O\left(\left|\lambda_{\sigma(j)}\right|+\left|\lambda_{\sigma(j+1)}\right|\right), \epsilon_{j}^{\sigma}=0(1)$ if $\left|\lambda_{\sigma(j)}\right|+\left|\lambda_{\sigma(j+1)}\right|$ is even (odd), $\epsilon_{\sigma(1)}=0(1)$ if $\left|\lambda_{\sigma(1)}\right|$ is odd (even), $\epsilon=0(1)$ if $\ell$ is odd (even) and the summation is over all separations $\left(\pi_{\lambda_{\sigma(1)}}, \pi_{2}^{\sigma}, \pi_{4}^{\sigma}, \ldots, \pi_{m-1}^{\sigma}\right)$ of $\pi$ corresponding to the composition.

Proof We prove (i) only; the proof of (ii) is similar. ¿From Theorem 3.5(i) and (4.16) we obtain

$$
\begin{gathered}
\sum_{\pi \in O(\ell)} 2^{\frac{1}{2}(m+\ell(\pi)+\epsilon)} z_{\pi}^{-1} \zeta_{\pi}^{\lambda} p_{\pi}(\mathbf{x}) \\
=\sum_{\sigma \in S_{m} / S_{2} l S_{\mu}} \epsilon(\mu) \prod_{i \in I} \sum_{\pi_{i}^{\sigma}} 2^{\frac{1}{2}\left(2+\ell\left(\pi_{i}^{\sigma}\right)+\epsilon_{i}^{\sigma}\right)} z_{\pi_{i}^{\sigma}}^{-1} \zeta_{\pi_{i}^{\sigma}}^{\left(\lambda_{\sigma(i)}, \lambda_{\sigma(i+1)}\right.} p_{\pi_{i}^{\sigma}}(\mathbf{x}) .
\end{gathered}
$$

Since, if $\left\{\pi^{1}, \pi^{2}, \pi^{3}, \ldots, \pi^{m}\right\}$ is a separation of $\pi \in O(\ell)$, we have $\ell(\pi)=\sum_{i=1}^{m} \ell\left(\pi^{i}\right), p_{\pi}(\mathbf{x})=$ $\prod_{i=1}^{m} p_{\pi_{i}}(\mathbf{x})$, and using (4.20) and comparing the coefficients of $p_{\pi}(\mathbf{x})$ on both sides of the above, we obtain the desired formula.

Remark 1: We note that simplifications of the above formula can be obtained in most cases; that is, in case (i)

$$
2^{\frac{1}{2}\left(-\epsilon+\sum_{i \in I} \epsilon_{i}^{\sigma}\right)}=1
$$

in the following cases:

(a) if $\ell$ is even and the parts of $\lambda$ are all even or are all odd,

(b) if $\ell$ is odd and all the parts of $\lambda$ except one are even or are odd,

and in case (ii)

$$
2^{\frac{1}{2}\left(\epsilon+\epsilon_{\sigma(1)}+\sum_{j \in J} \epsilon_{j}^{\sigma}\right)}=1
$$

in the following cases

(a) if $\ell$ is even and all the parts of $\lambda$ are either even or are odd except $\lambda_{\sigma(1)}$,

(b) if $\ell$ is odd and all the parts of $\lambda$ are either odd or are even except for $\lambda_{\sigma(1)}$. 
Furthermore, in order to apply these formulae, we require to determine left coset representatives of $S_{2} S_{\mu}$ in $S_{m}$ for both $m=2 \mu$ and $2 \mu+1$. We note that

$$
\left[S_{m}: S_{2} S_{\mu}\right]=\left\{\begin{array}{c}
1 \cdot 3 \cdot \ldots m-1 \\
1 \cdot 3 \cdot \ldots \cdot m
\end{array} \quad \text { if } m=2 \mu\right. \text { is even, }
$$

If $m=2 \mu$ is even, left coset representatives of $S_{2} \succ S_{\mu}$ in $S_{m}$ are of the form $\psi=x_{1} x_{2} \ldots x_{m-1}$, where $x_{i}=\left(x_{i 1}, x_{i 2}\right)$ are transpositions determined inductively as follows:

(i) Put $I_{1}=\{2, \ldots, m\}$, then $x_{11}=2, x_{12}=k_{1}, k_{1} \in I_{1}$.

(ii) Put $I_{2}=I \backslash\left\{x_{11}, x_{12}\right\}, d_{2}=\min I_{2}$, then $x_{21}=d_{2}, x_{22}=k_{2}, k_{2} \in I_{2}$ unless $k_{1}=2$, in which case $x_{21}=d_{2}+1$.

(iii) Put $I_{r}=I_{r-1} \backslash\left\{x_{r-1,1}, x_{r-1,2}\right\}, d_{r}=\min I_{r}$, then $x_{r 1}=d_{r}, x_{r 2}=k_{r}, k_{r} \in I_{r}$, unless $k_{r-1}=d_{r-1}$, in which case $x_{r 2}=d_{r-1}+1$.

For example, the left coset representatives of $S_{2} \prec S_{3}$ in $S_{6}$ are given as follows

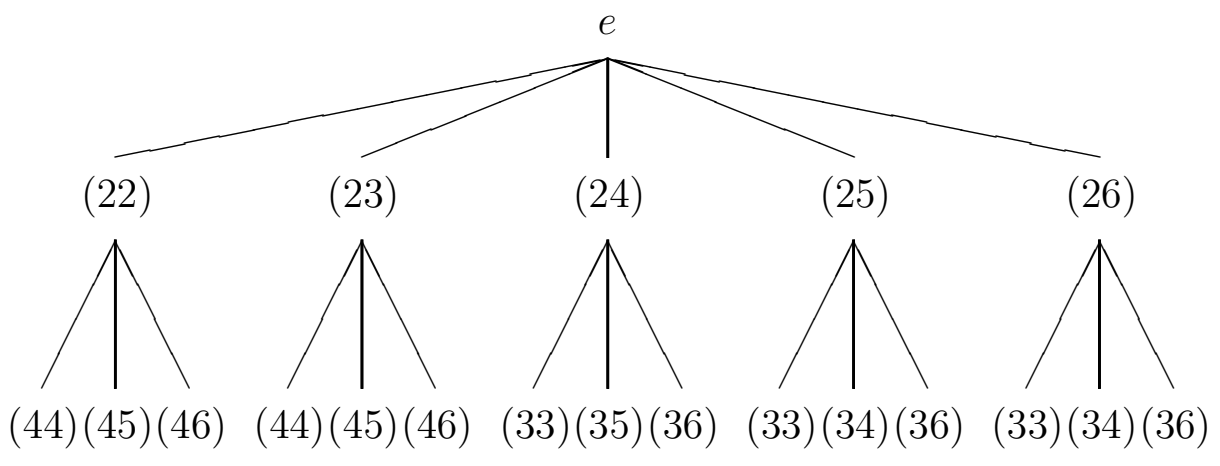

that is, $\quad$ e, (45), (46), (23), (23)(45), $\quad(23)(46), \quad(24), \quad(24)(35), \quad(24)(36), \quad(25)$, (25)(34), (25)(36), (26), (26)(34), (26)(35)\}.

Similarly, if $m=2 \mu+1$ is odd, the left coset representatives of $S_{2}$ 乙 $S_{\mu}$ in $S_{m}$ are of the form $\phi=\phi_{1} \psi$, where $\phi_{1}$ can be any of the $m$ transpositions $(1, k)(1 \leq k \leq m)$ and $\psi$ is obtained in exactly the same way as the above but now applied to the set $J=\{3,4, \ldots, m=2 \mu+1\}$ in place of $I=\{2, \ldots, 2 \mu\}$. Thus, for example, the left coset representatives of $S_{2} S_{2}$ in $S_{5}$ are $\{e,(12),(13),(14),(15)\} \times\{e,(34),(35)\}=\{e,(34),(35), 12),(12)(34),(12)(35),(13),(13)(34)$, (13)(35),(14), (14)(34), (14)(35), (15), (15)(34), (15)(35)\}.

Example 1: The values of the character $\zeta^{(6,4,3,2)}$ of $S_{15}$ are calculated at the classes $\left(1^{2} 3^{2} 7\right)$ and $(3,5,7)$. In this case, as $\left(\lambda_{1}, \lambda_{2}, \lambda_{3}, \lambda_{4}\right)=(6,4,3,2)$, the relevant left coset representatives are $e,(23),(24)$; thus, by Theorem $4.2(\mathrm{i})$ and Remark 1(i), we have

$$
\begin{gathered}
\zeta_{\left(1^{2} 3^{2} 7\right)}^{(6,4,3,2)}=\frac{2 ! 2 !}{2 !} \zeta_{(3,7)}^{(6,4)} \zeta_{\left(1^{2} 3\right)}^{(3,2)}-\frac{2 ! 2 !}{2 ! 2 !} \zeta_{\left(1^{2} 7\right)}^{(6,3)} \zeta_{\left(3^{2}\right)}^{(4,2)} \\
+\frac{2 ! 2 !}{2 !} \zeta_{(1,7)}^{(6,2)} \zeta_{\left(1,3^{2}\right)}^{(4,3)}+\frac{2 ! 2 !}{2 ! 2 !} \zeta_{\left(1^{2} 3^{2}\right)}^{(6,2)} \zeta_{(7)}^{(4,3)}
\end{gathered}
$$


noting that $\left((3,7),\left(1^{2} 3, \phi\right)\right)$ and $\left(\left(1^{2} 7, \phi\right),\left(3^{2}, \phi\right)\right)$ are the only separations of $\left(1^{2} 3^{2} 7\right)$ corresponding to $((6,4),(3,2))$ and $((6,3),(4,2))$ respectively, while there are two separations $\left((1,7),\left(13^{2}, \phi\right)\right)$ and $\left(\left(1^{2} 3^{2}\right), \phi\right),((7), \phi)$ of $\left(1^{2} 3^{2} 7\right)$ corresponding to $((6,2),(4,3,1))$.

Now by means of Theorem 4.1, we find that

$$
\begin{gathered}
\zeta_{(3,7)}^{(6,4)}=0, \quad \zeta_{\left(1^{2}, 3\right)}^{(3,2)}=-1, \quad \zeta_{\left(1^{2} 7\right)}^{(6,3)}=0, \quad \zeta_{\left(3^{2}\right)}^{(4,2)}=2, \quad \zeta_{(17)}^{(6,2)}=0 \\
\zeta_{\left(13^{2}\right)}^{(4,3)}=2, \quad \zeta_{\left(1^{2} 3^{2}\right)}^{(6,2)}=-2, \quad \zeta_{(7)}^{(4,3)}=-1 .
\end{gathered}
$$

Thus

$$
\zeta_{\left(1^{2} 3^{2} 7\right)}^{(6,4,3)}=\zeta_{\left(1^{2} 3^{2}\right)}^{(6,2)} \zeta_{(7)}^{(4,3)}=2 .
$$

Since the partition $(3,5,7)$ has no separations corresponding to $\pi_{9} \in O(9), \pi_{6} \in O(6)$, we have

$$
\begin{gathered}
\zeta_{(3,5,7)}^{(6,4,3,2)}=\zeta_{(3,7)}^{(6,4)} \zeta_{(5)}^{(3,2)}+\zeta_{(3,5)}^{(6,2)} \zeta_{(7)}^{(4,3)} \\
=0 \cdot 1-2 \cdot(-1)=+2
\end{gathered}
$$

Example 2: The values of the character $\zeta^{(5,4,3,2,1)}$ of $S_{15}$ are calculated at the classes $\left(1^{2} 3^{2} 7\right)$ and $(3,5,7)$. In this case, there are terms corresponding to the 15 left coset representatives of $S_{2} \prec S_{2}$ in $S_{5}$ listed above. However, since $\zeta_{(1,7)}^{(5,3)}=\zeta_{\left(1^{2} 7\right)}^{(5,4)}=\zeta_{\left(1^{2} 3\right)}^{(4,1)}=\zeta_{\left(13^{2}\right.}^{(5,2)}=0$, then by Theorem $4.2\left(\right.$ ii) and Remark 1(ii) and taking the only possible separations of $\left(1^{2} 3^{2} 7\right)$ we obtain

$$
\begin{gathered}
\zeta_{\left(1^{2} 3^{2} 7\right)}^{(5,4,3,2,1)}=\frac{2 ! 2 !}{2 !} \cdot 2 \zeta_{\left(1^{2} 3\right)}^{(5)} \zeta_{(7)}^{(4,3)} \zeta_{(3)}^{(2,1)}+\frac{2 ! 2 !}{1} \cdot 2 \cdot \zeta_{(1,3)}^{(4)} \zeta_{(7)}^{(5,2)} \zeta_{(1,3)}^{(3,1)} \\
-\left(-\frac{2 ! 2 !}{2 ! 2 !} \cdot 2 \zeta_{\left(1^{2}\right)}^{(2)} \zeta_{\left(1^{2}\right)}^{(2)} \zeta_{\left(3^{2}\right)}^{(5,1)} \zeta_{(7)}^{(4,3)}\right)+\frac{2 ! 2 !}{2 !} \cdot 2 \cdot \zeta_{(1)}^{(1)} \zeta_{(7)}^{(5,2)} \zeta_{\left(13^{2}\right)}^{(4,3)}
\end{gathered}
$$

Again, by Theorem 4.1, we have $\zeta_{(7)}^{(4,3)}=-1, \zeta_{(3)}^{(2,1)}=-1, \zeta_{(7)}^{(5,2)}=1, \zeta_{(1,3)}^{(3,1)}=-(-1), \zeta_{\left(3^{2}\right)}^{(5,1)}=-2$ and $\zeta_{\left(13^{2}\right)}^{(4,3)}=2$, and by $(4.17)$, we have $\zeta_{\left(1^{2} 3\right)}^{(5)}=2, \zeta_{(13)}^{(4)}=1, \zeta_{\left(1^{2}\right)}^{(2)}=1$ and $\zeta_{(1)}^{(1)}=1$. Thus, we have

$$
\zeta_{\left(1^{2} 3^{2} 7\right)}^{(5,3,2,1)}=4 .
$$

Due to the separation process, there are only two terms to be considered for the class $(3,5,7)$ and we obtain

$$
\begin{aligned}
\zeta_{(3,5,7)}^{(5,4,3,2,1)} & =2 \zeta_{(5)}^{(5)} \zeta_{(7)}^{(4,3)} \zeta_{(3)}^{(2,1)}-2 \zeta_{(3)}^{(3)} \zeta_{(7)}^{(5,2)} \zeta_{(5)}^{(4,1)} \\
& =2 \cdot 1 \cdot(-1) \cdot(-1)-2 \cdot 1 \cdot 1 \cdot(-1)=4
\end{aligned}
$$

\section{References}

[1] P.N. Hoffman and J.F. Humphreys: Projective Representations of the Symmetric Groups Q-functions and Shifted Tableaux, Clarendon Press, Oxford, 1992.

[2] G.D. James and A. Kerber: The Representation Theory of the Symmetric Group, AddisonWesley, Reading, Mass., 1981.

[3] D.E. Littlewood and A.R. Richardson: Group characters and algebras, Phil. Trans. Roy. Soc. London, Ser A 233 (1934). 
[4] I.G. Macdonald: Symmetric Function and Hall Polynomials, Clarendon Press, Oxford, 1979.

[5] A.O. Morris: The spin representations of the symmetric group, Proc. London Math. Soc. (3), 12 (1962), 55-76.

[6] I. Schur: Über die Darstellungen der symmetrischen und der alternierenden Gruppe durch gebrochen lineare Substituionen, J. für Math. 139 (1911), 155-250. 